\title{
Localisation and direction of mitral regurgitant flow in mitral orifice studied with combined use of ultrasonic pulsed Doppler technique and two dimensional echocardiography
}

\author{
KUNIO MIYATAKE, YASUHARU NIMURA, HIROSHI SAKAKIBARA, \\ NAOKAZU KINOSHITA, MITSUNORI OKAMOTO, SEIKI NAGATA, KOHEI KAWAZOE, \\ TSUYOSHI FUJITA
}

From the National Cardiovascular Centre, Department of Cardiology, and the Research Institute, Osaka, Fapan

SUMMARY Regurgitant flow was analysed in 40 cases of mitral regurgitation, using combined ultrasonic pulsed Doppler technique and two dimensional echocardiography.

Abnormal Doppler signals indicative of mitral regurgitant flow were detected in reference to the two dimensional image of the long axis view of the heart and the short axis view at the level of the mitral orifice.

The overall direction of regurgitant flow into the left atrium was clearly seen in 28 of 40 cases, and the localisation of regurgitant flow in the mitral orifice in 38 cases.

In cases with mitral valve prolapse of the anterior leaflet or posterior leaflet the regurgitant flow was directed posteriorly or anteriorly, respectively. The prolapse occurred at the anterolateral commissure or posteromedial commissure and resulted in regurgitant flow located near the anterolateral commissure or posteromedial commissure of the mitral orifice, respectively. In cases with rheumatic mitral regurgitation the regurgitant flow is usually towards the central portion of the left atrium and is sited in the mid-part of the orifice. The Doppler findings were consistent with left ventriculography and surgical findings.

The ultrasonic pulsed Doppler technique combined with two dimensional echocardiography is useful for non-invasive analysis and preoperative assessment of mitral regurgitation.

With the development of the ultrasonic pulsed Doppler technique, 12 the conditions of blood flow in the cardiac cavities in cases of valvular and congenital heart diseases have been investigated and progress is being made in clinical application..$^{3-6}$ We have already studied cases of mitral regurgitation using a combination of the ultrasonic pulsed Doppler technique and two dimensional echocardiography and have reported that abnormal Doppler signals interpreted to indicate regurgitant flow were detected in the left atrial cavity. ${ }^{6}$ In the present study, the direction of the regurgitant flow into the left atrial cavity and localisation of the regurgitant flow in the mitral orifice were studied. A

Supported in part by the Scientific Research Grants for Health and Welfare programme of the Ministry of Health and Welfare of Japan in 1981, and Grant in Aid for Development Scientific Research from the Ministry of Education, Science and Culture of Japan in 1979 and 1980.

Accepted for publication 15 June 1982 comparative study was also made on the relation between these conditions and the pathological changes in the mitral valve.

\section{Subjects and methods}

Forty patients with mitral regurgitation resulting from various underlying conditions were examined in our laboratory during one year from April 1979 to March 1980. The ages of the patients ranged from 28 to 65 years, with an average of 42 . The underlying disease in these $\mathbf{4 0}$ cases of mitral regurgitation was rheumatic valvular disease in 26 cases, idiopathic mitral valve prolapse in 11 cases, subacute bacterial endocarditis in two cases, and severe dilatation of the left ventricle caused by aortic regurgitation in one case.

Rupture of the chordae tendineae of the mitral valve was also found in eight of the 11 cases of idiopathic 
mitral valve prolapse and in one of the two cases of subacute bacterial endocarditis. Prolapse of the mitral valve or rupture of the chordae tendineae were seen in the anterior leaflet of the mitral valve in six cases, in the posterior leaflet in three cases, and in both leaflets in three cases. These 40 cases were all diagnosed by routine clinical examinations, $M$-mode and real time two dimensional echocardiography, and biplane left ventriculography.

Thirty four of the 40 subjects subsequently underwent mitral valve replacement or mitral valve repair. Ten healthy subjects were also examined as controls.

The equipment used was a combination of a directional ultrasonic pulsed Doppler flowmeter (Hitachi Medico EUD-4Z) and a two dimensional echocardiograph (Aloka, SSD-120). The pulsed Doppler flowmeter had a $2.5 \mathrm{MHz}$ transducer and there were two pulse repetition rates, 4.5 and $6.3 \mathrm{KHz}$. The size of the sample volume was $6 \times 6 \times 2 \mathrm{~mm}^{3}$ or $6 \times 6 \times 5 \mathrm{~mm}^{3}$. The Doppler examination was performed by monitoring the sampling site on the two dimensional echocardiogram with this equipment (Fig. 1). First, the ultrasound beam was transmitted from the third or fourth intercostal space of the left sternal border into the left atrium. A search for the abnormal Doppler signals was carried out over the mitral orifice and the left atrial cavity. If there were abnormal Doppler signals indicating mitral regurgitant flows, the two dimensional echocardiogram along the long axis of the left ventricle was recorded including the beam direction in which these abnormal Doppler signals were detected. Secondly, the Doppler ultrasound beam was transmitted in the left atrium on this two dimensional cross sectional plane; the segmental range in this beam direction, where the $\overrightarrow{\vec{P}}$ abnormal regurgitant signals were detected, was $\frac{7}{0}$ determined by moving the sample volume continuously on this beam. Then, this beam direction and both ends $\bar{c}$ of the segment where the regurgitant flow signals were $\overrightarrow{\mathbb{D}}$ detected were superimposed on the two dimensional $\propto$ image as white line and white dots, respectively. क Changing the directions of the Doppler beam succes- $\overrightarrow{0}$ sively over the mitral orifice and the left atrium, the $\vec{\overrightarrow{ }}$ segments on each beam direction where the regurgitant $\tilde{\sigma}$ signals were detected were recorded as described above. Consequently, the distribution of the mitral regurgitant signals in the cross sectional plane was $\stackrel{+}{\infty}$ shown as the area encircled by the white dots (Fig. 1).

Next, the short axis cross section of the mitral orifice $\frac{\vec{t}}{0}$ was recorded. The first step in the setting was to produce 0 a cross section through the left atrial cavity just above the mitral orifice, which was almost perpendicular to $\bar{z}$ the long axis cross section. The short axis cross section was then shifted as near the mitral orifice as possible, involving the range where the abnormal Doppler signals were detected.

The Doppler signals underwent real time frequency $\vec{\oplus}$ analysis. The instantaneous flow velocity was continuously displayed in reference to the time axis. Here, $\square$ the flow components towards the transducer were dis-? played below the baseline and those away from the transducer above the baseline. The scale markers of the frequency are shown in Fig. 2 as $1 \mathrm{KHz}$ and $2 \mathrm{KHz}$. Ф The scale of $1 \mathrm{KHz}$ frequency meant the velocity of $30 \stackrel{2}{\Rightarrow}$ $\mathrm{cm} / \mathrm{s}$ along the beam direction. All the figures were $\frac{0}{3}$ recorded in reference to the same frequency scale. These flow velocity spectrograms were simultaneouslyo

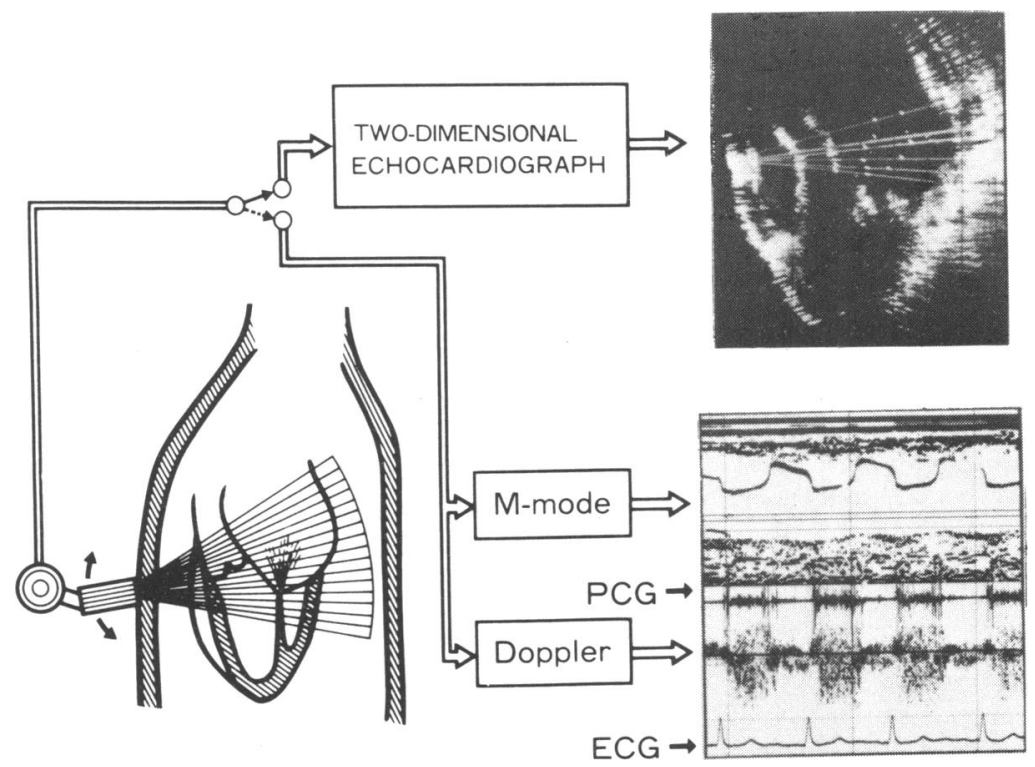

Fig. 1 Diagram of the detection of mitral regurgitation by the combined use of the ultrasonic pulsed Doppler flowmeter and two dimensional echocardiography. Upper right panel: two dimensional echocardiogram along the long axis of the heart. White lines indicate the beam directions for the Doppler technique. The distribution of the abnormal Doppler signals is shown by the area encircled by the white dots. Lower right panel: simultaneous recording of Doppler signals with the $N$. mode echocardiogram, phonocardiogra (PCG), and electrocardiogram (ECG) 

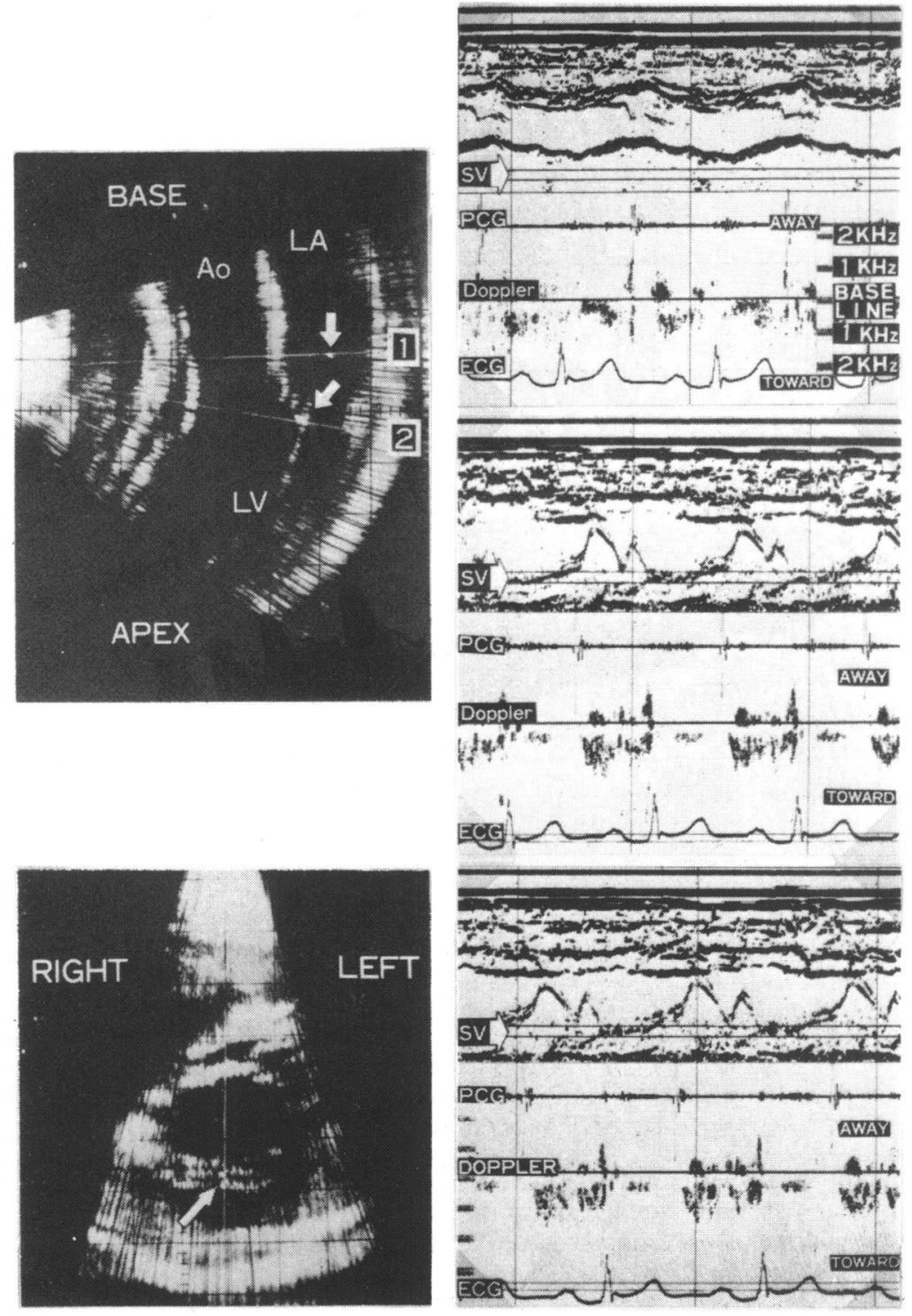

Fig. 2 Intracardiac Doppler signals in a healthy 29 year old man. Upper right panel: the Doppler signals in the left atrial cavity. Middle right panel: the Doppler signals in the mitral orifice. The upper right panel was recorded along the line 1 and the middle right panel along the line 2 in the long axis image of the heart on the upper left panel. Arrows in the two dimensional image indicate the positions of the sample volume (SV) of the Doppler signal. Lower right panel: the Doppler signals were recorded at the site indicated by the white arrow in the short axis image at the level of the mitral orifice on the lower left panel. Ao, aorta; $L A$, left atrium; $L V$, left ventricle. recorded with the $M$-mode echocardiogram by the same beam; the electrocardiogram and the phonocardiogram were recorded at the upper sternal area. The site of the sample volume was also indicated on the M-mode echocardiogram (Fig. 2).

\section{Results}

\section{HEALTHY SUBJECTS}

In systole, slow flow signals toward the transducer were recorded in the left atrial cavity. In the mitral orifice only a few Doppler signals based on the movement of the mitral valve were recorded (Fig. 2). These findings were the same as those in the preliminary report. ${ }^{6}$

\section{MITRAL REGURGITATION}

(1) General aspect

In cases with mitral regurgitation, abnormal Doppler signals which were pansystolic and showed a wide band velocity spectrum were detected in the left atrial cavity and the mitral orifice. The spectrum consisted of unidirectional or bidirectional components. As reported previously, ${ }^{6}$ these signals were considered to indicate 

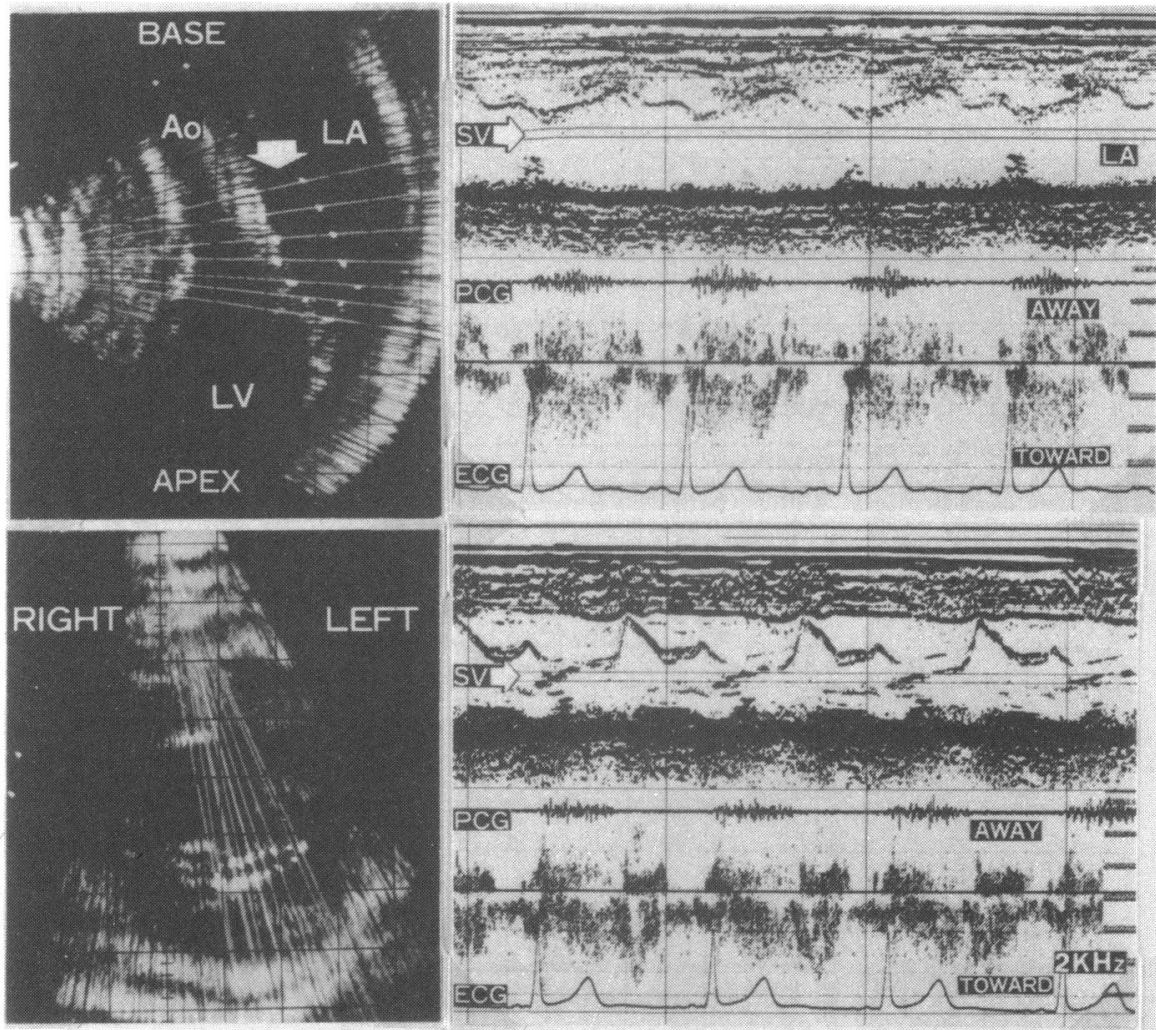

Fig. 3 Abnormal Doppler signals in a 32 year old woman with idiopathic mitral valve prolapse of the posterior mitral leaflet in the anterolateral commissure. Upper right and lower right panels: the abnormal Doppler signals were detected in the left atrial cavity and in the mitral orifice, respectively. Upper left and lower left panels: the distributions of the abnormal Doppler signals are shown by the area encircled by white dots in the left atrial cavity and the mitral orifice, respectively. The regurgitant flow was directed to the anterior along the anterior mitral leaflet and the anterior wall of the left atrium. In the mitral orifice, the regurgitant flow was localised near the anterolateral commissure. Abbreviations as in Fig. 2.

the turbulence of the mitral regurgitant flow, and low pitch powerful signals at the mitral orifice were considered to indicate the regurgitant flow and the valve vibration caused by the regurgitant flow. The directions of the flow components in the sample volume were not necessarily interpreted to indicate the overall direction of the regurgitant flow, but the flow directions in the turbulence in the regurgitant flow. The overall direction of the regurgitant flow was considered to be indicated by the spatial orientation of the range in the left atrial cavity on the long axis image, where the abnormal Doppler signals were detected. The range of the abnormal Doppler signals at the mitral orifice on the short axis image was interpreted to indicate the regurgitant part of the mitral orifice.

Example 1: A 32 year old woman had idiopathic mitral valve prolapse of the posterior leaflet at the anterolateral commissure.

The abnormal Doppler signals were detected in the anterior part of the left atrial cavity on the long axis image and at the lateral half of the mitral orifice on the short axis image (Fig. 3). These findings were interpreted to mean that the mitral regurgitant flow occurred near the anterolateral commissure of the mitral orifice and was directed anteriorly.

Example 2: A 29 year old woman had rheumatic mitral regurgitation with dislocation of the coaptation edge of the anterior mitral leaflet into the left atrial cavity.

The abnormal Doppler signals were detected in the posterior part of the left atrial cavity on the long axis image and at the mid-part of the mitral orifice (Fig. 4), probably indicating that the mitral regurgitant flow occurred at the mid-part of the mitral orifice and was directed posteriorly.

(2) Direction of regurgitant flow into left atrial cavity

The overall directions of the regurgitant flow from the mitral orifice into the left atrial cavity were clearly 

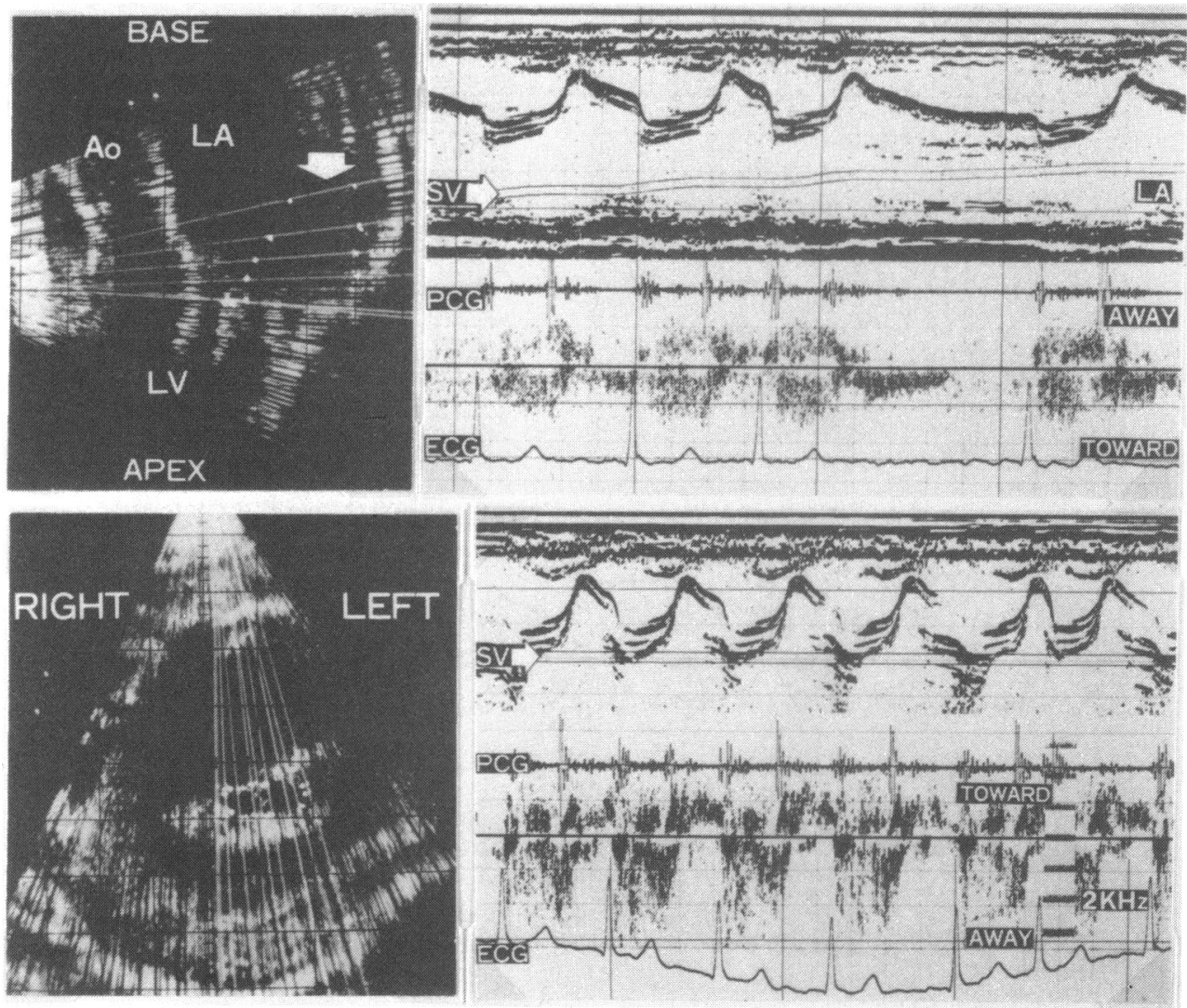

Fig. 4 Abnormal Doppler signals in a 29 year old woman with rheumatic mitral regurgitation. Upper right and lower right panels: the abnormal Doppler signals were detected in the left atrial cavity and the mitral orifice, respectively. Upper left panel: the abnormal Doppler signals were distributed from the mitral orifice to the posterior half of the left atrial cavity along the posterior wall of the left atrium. Lower left panel: the abnormal Doppler signals were localised in the mid-part of the mitral orifice. Abbreviations as in Fig. 2.

recorded in 28 out of 40 cases and could be classified into the following three groups (Table 1 )

(i) The regurgitant flow signals were detected in the anterior part of the left atrial cavity along the anterior wall of the left atrium (Fig. 3). Such findings were thought to indicate that the regurgitant flow was directed toward the anterior portion of the left atrial cavity, and they were obtained in four of the 28 cases. Three of these four cases had idiopathic prolapse of the posterior mitral leaflet, and the remaining case had idiopathic mitral valve prolapse predominantly in the posterior leaflet though the anterior leaflet showed slight prolapse.

(ii) The regurgitant flow signals were detected in the posterior part of the left atrial cavity along the posterior wall of the left atrium (Fig. 4). This distribution of the regurgitant flow signals was interpreted to indicate that the regurgitant flow was directed toward the posterior portion of the left atrial cavity, and such findings were
Table 1 Direction of mitral regurgitation in left atrial cavity and site of mitral regurgitation in orifice estimated by Doppler method in 38 cases

\begin{tabular}{|c|c|c|c|}
\hline \multirow{3}{*}{$\begin{array}{l}\text { Overall direction of } \\
\text { regurgitant flow in } \\
\text { left atrial cavity }\end{array}$} & \multicolumn{3}{|c|}{ Site of regurgitant flow in the mitral orifice } \\
\hline & $\begin{array}{l}\text { Posteromedial } \\
\text { part }\end{array}$ & $\begin{array}{l}\text { Mid-part or } \\
\text { wide }\end{array}$ & $\begin{array}{l}\text { Anterolateral } \\
\text { part }\end{array}$ \\
\hline & No. of cases & No. of cases & No. of cases \\
\hline $\begin{array}{l}\text { Anterior portion } \\
\text { Central portion or } \\
\text { widely distributed }\end{array}$ & $\begin{array}{l}0 \\
1\end{array}$ & $\begin{array}{r}2 \\
14\end{array}$ & $\begin{array}{l}2 \\
0\end{array}$ \\
\hline $\begin{array}{l}\text { Posterior portion } \\
\text { Unidentified }\end{array}$ & $\begin{array}{l}3 \\
1\end{array}$ & $\begin{array}{l}4 \\
9\end{array}$ & $\begin{array}{l}2 \\
0\end{array}$ \\
\hline
\end{tabular}

obtained in nine cases. Three of these nine cases had idiopathic prolapse of the anterior mitral leaflet. One case had prolapse in both leaflets, dominant in the anterior leaflet. Three of the above nine cases had rheumatic valvular disease and two had subacute 

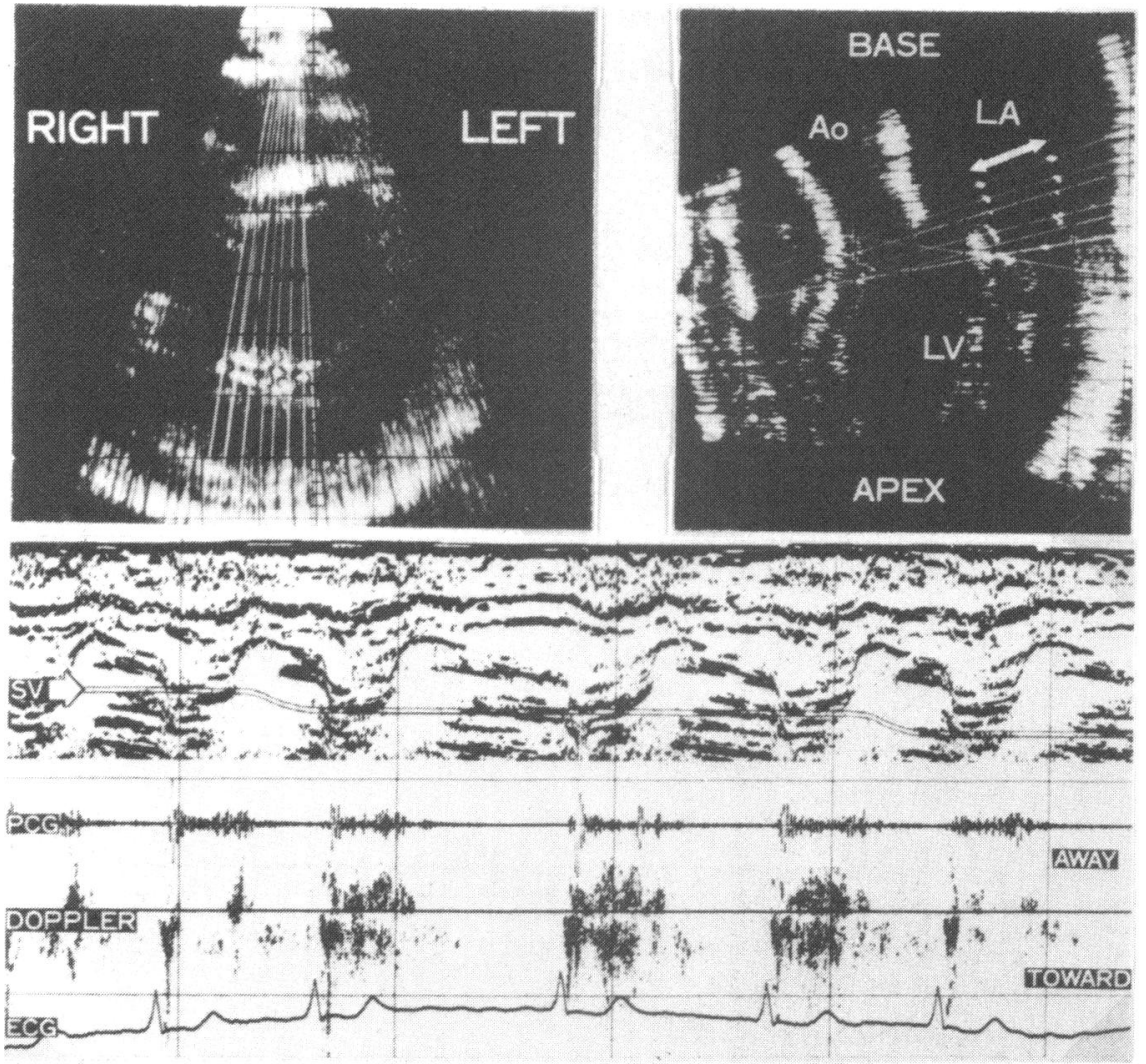

Fig. 5 Abnormal Doppler signals in a 44 year old woman with rheumatic mitral regurgitation. Upper right panel: the long axis section of the heart. The abnormal Doppler signals were found in the central portion of the left atrial cavity extending from the mitral orifice. Upper left panel: the short axis section at the level of the mitral orifice. The abnormal Doppler signals were found in the area, slit-like in shape, in the mid-part of the orifice. Lower panel: the abnormal Doppler signals were recorded in the mitral orifice.

Abbreviations as in Fig. 2.

bacterial endocarditis. In one of the cases of rheumatic valvular disease, a dislocation of the coaptation edge of the anterior mitral leaflet into the left atrial cavity was disclosed at operation and in one of the cases with subacute bacterial endocarditis a ruptured chorda of the anterior mitral leaflet was also shown at operation.

(iii) The regurgitant flow signals were detected in the central portion of the left atrial cavity (Fig. 5) or widely over the left atrial cavity. Such findings were interpreted to indicate that the overall direction of regurgitant flow was mainly directed toward the central portion of the left atrial cavity. Such regurgitant flows as were sited in the anterior half or posterior half of the left atrial cavity were also included in this group only if they were separated from the anterior or posterior wall of the left atrium (Fig. 6). These findings were noted in 15 cases, 13 of whom had rheumatic valvular disease. The other two had prolapse of the anterior leaflet or both leaflets.
These three groups of regurgitant flow obtained by the Doppler method were compared with the group obtained by left ventriculography (Table 2 ). The overall direction of regurgitant flow detected by the Doppler method was similar to the direction of flow found by left ventriculography in 27 out of 28 cases.

Table 2 Comparison between direction of mitral regurgitant flow estimated by Doppler method and that by left ventriculography in 28 cases

\begin{tabular}{llll}
\hline Left ventriculography & \multicolumn{2}{l}{ Doppler method } \\
\cline { 2 - 3 } & Anterior & $\begin{array}{c}\text { Central portion or } \\
\text { widely distributed }\end{array}$ & Posterior \\
\cline { 2 - 3 } & \multicolumn{2}{l}{$\frac{\text { No. of cases }}{\text { No. of cases }}$} & No. of cases \\
\hline $\begin{array}{llll}\text { Anterior } \\
\begin{array}{l}\text { Central portion or } \\
\text { widely distributed }\end{array}\end{array}$ & 4 & 0 & 0 \\
Posterior & 0 & 14 & 0 \\
\hline
\end{tabular}




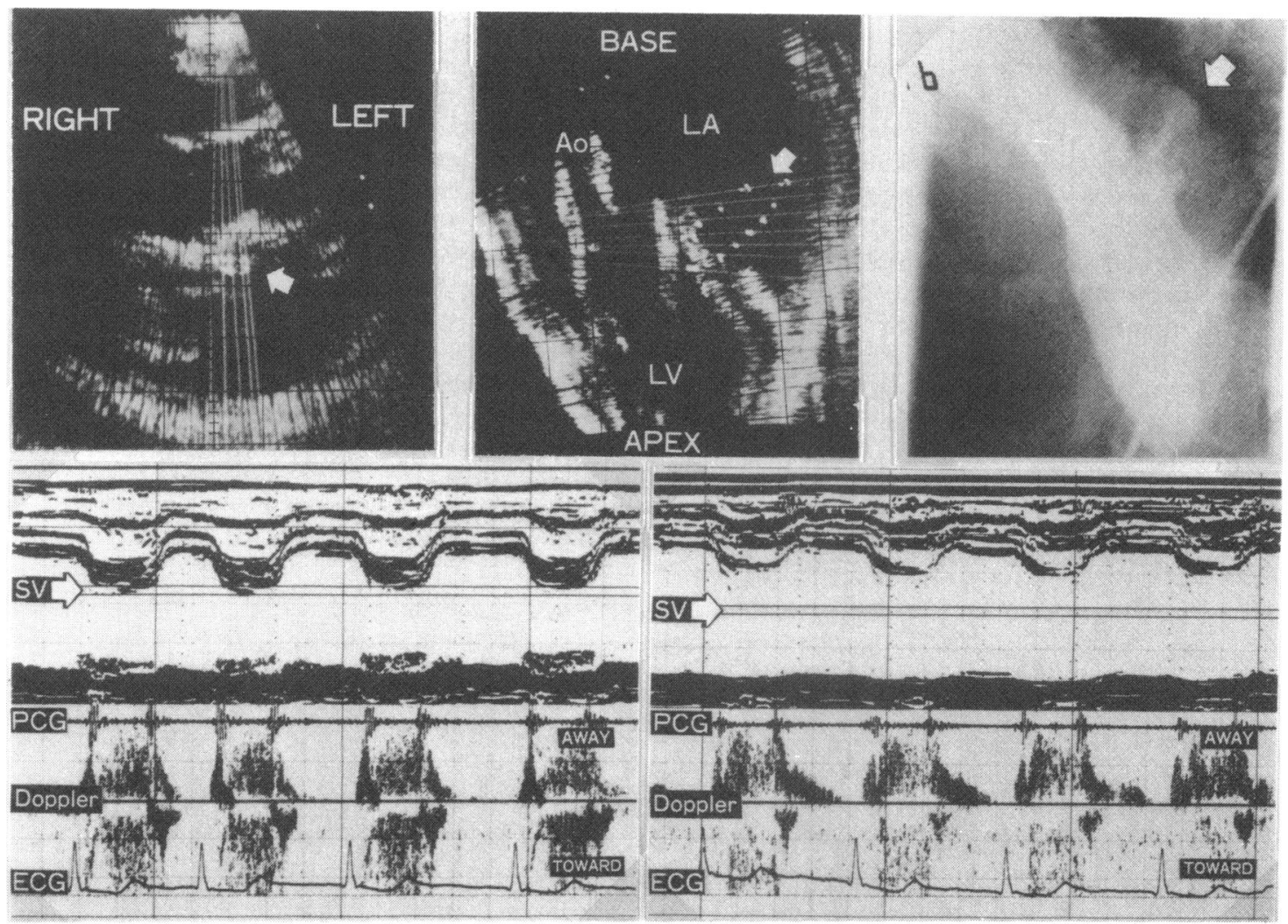

Fig. 6 Abnormal Doppler signals in a 53 year old woman with rheumatic mitral stenosis and regurgitation. Upper middle panel: the long axis section of the heart showing the distribution of the abnormal Doppler signals directed from the mitral orifice to the posterior. In this patient, however, the abnormal Doppler signals were not detected along the posterior wall of the left atrium. Upper right panel: left lateral view of the left ventriculogram. The direction of the regurgitant flow detected by the Doppler method was fairly consistent with that shown by left ventriculography. Upper left panel: short axis section at the level of the mitral orifice. The abnormal Doppler signals were found in the small area, at the mid-part of the orifice. Lower right and lower left panels: the abnormal Doppler signals were detected in the left atrial cavity and in the mitral orifice, respectively. Abbreviations as in Fig. 2.

There was one exception, a case of ruptured chordae tendineae of the anterior mitral leaflet, where the regurgitant flow was found toward the central portion of the left atrial cavity by the Doppler method, while it was shown directed toward the posterior portiof of the left atrial cavity on left ventriculography.

(3) Localisation of regurgitant flow in mitral orifice

In the short axis image of the mitral orifice, abnormal systolic Doppler signals were found along the coaptation line of the anterior and posterior leaflets of the mitral valve in $\mathbf{3 8}$ out of $\mathbf{4 0}$ cases. These abnormal signals had powerful bidirectional components, with a wide band" of velocity spectrum.

The range of detection of these abnormal Doppler signals was localised in a small spot on or in a narrow band along the coaptation line. The extent of this range and the location in the mitral orifice varied in accordance with the case. The location of the detecting range could be roughly divided into the following three sites (Table 1).

(i) The regurgitant flow signals were observed in the range from the posteromedial commissure to the midpart of the mitral orifice (but not beyond the mid-part of the mitral orifice) (Fig. 7). These findings were noted in five of the 38 cases. Three of these five cases had mitral valve prolapse of the posteromedial commissure, one had ruptured chordae tendineae of the posteromedial commissure caused by subacute bacterial endocarditis, and the other case had rheumatic valvular disease.

(ii) The regurgitant flow signals were observed in the range from the anterolateral commissure to the midpart of the mitral orifice (but not beyond the mid-part of the mitral orifice) (Fig. 3). Such signals were found in four of the 38 cases. Two of these four cases had idiopathic mitral valve prolapse of the anterolateral 


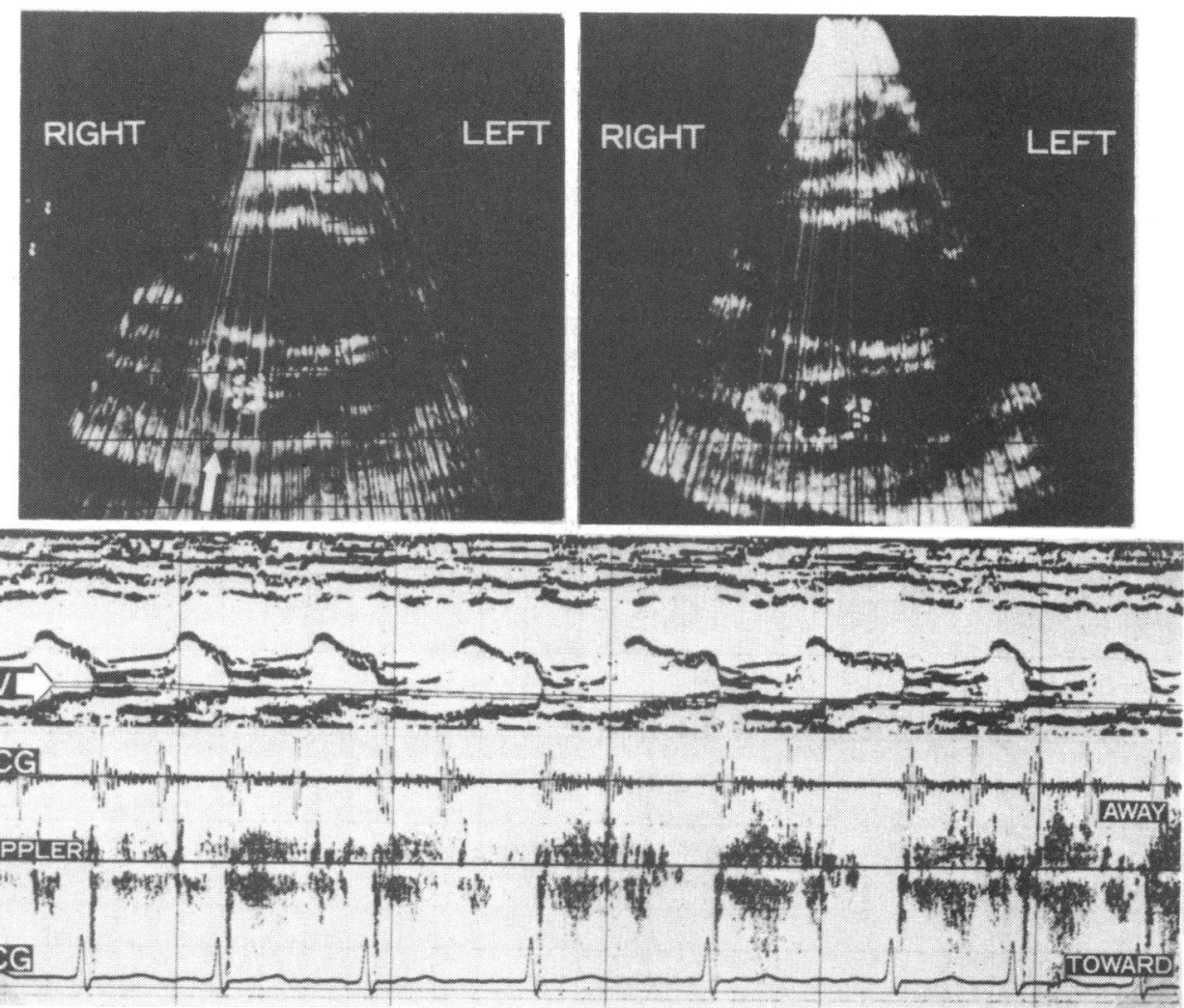

Fig. 7 Abnormal Doppler signals in a 54 year old woman with mitral valve prolapse of the anterior mitral leaflet at the posteromedial commissure. Upper left panel: short axis section at the level of the mitral orifice. The abnormal Doppler signals were found near the posteromedial commissure of the orifice. Upper right panel: short axis section slightly superior to the orifice. In this section, the distribution of the abnormal Doppler signals became wide and shifted posteriorly as compared with that at the mitral orifice level as shown in the upper left panel. This indicates that the regurgitant flow was directed posteriorly. Lower panel: the abnormal Doppler signals in the mitral orifice were recorded in the beam direction indicated by the arrow in the upper left panel. Abbreviations as in Fig. 2.

commissure, one had, rheumatic valvular disease, and one had subacute bacterial endocarditis.

(iii) The regurgitant flow signals were found in the mid-part of the mitral orifice (Fig. 5) or over a wide range along the coaptation line of the mitral valve. This was observed in 29 cases, of whom 22 had rheumatic valvular disease, six had idiopathic mitral valve prolapse, and one had dilatation of the left ventricle.

These three sites of regurgitant flow in the mitral orifice detected by the Doppler method were compared with those assumed from the valve lesions seen during heart surgery (Table 3 ).

The morphological changes in the valve were examined at operation in 34 of the 38 cases in whom abnormal Doppler signals were detected at the mitral orifice. In 31 of the 34 cases, there was agreement between the findings obtained by the Doppler method and those seen during heart surgery. The sites of val- vular lesions, such as mitral valve prolapse, ruptured chordae tendineae, or rheumatic fusion of the leaflets, seen at operation corresponded to those assumed from Doppler findings, that is at the posteromedial part of the mitral orifice in five cases, at the mid-part in 24 cases, and at the anterolateral part in two cases.

Table 3 Comparison between localisation of mitral regurgitant flow in orifice estimated by Doppler method and that at operation in 34 cases

\begin{tabular}{llll}
\hline Surgery & \multicolumn{2}{l}{ Doppler method } & \\
\cline { 2 - 3 } & Posteromedial & $\begin{array}{l}\text { Mid-part or } \\
\text { wide }\end{array}$ & Anterolateral \\
\cline { 2 - 2 } & No. of cases & No. of cases & No. of cases \\
\hline $\begin{array}{llll}\text { Posteromedial } \\
\text { Mid-part or wide }\end{array}$ & 5 & 2 & 0 \\
Anterolateral & 0 & 0 & 1 \\
\hline
\end{tabular}


There were, however, two exceptions to this general correspondence. One was a case of rheumatic valvular disease, where regurgitant flow signals were detected in the anterolateral part of the mitral orifice; at operation, however, the mitral valve was fused at both commissures and the site of regurgitation was assumed to be in the mid-part of the orifice rather than in the anterolateral part. The other exception was in two cases of idiopathic mitral valve prolapse, where regurgitant flow signals were detected in the mid-part of the orifice by the Doppler method, while the surgical findings showed prolapse in the posteromedial commissure.

\section{Discussion}

With the progress of real-time two dimensional echocardiography, many new findings have been accumulated concerning changes in valvular morphology and haemodymanics in mitral valve disease and these results have been widely applied clinically. ${ }^{7}$ It is difficult, however, for echocardiography to detect direct findings of mitral regurgitation. ${ }^{8}$ For the direct diagnosis it is necessary to detect regurgitant flow. The ultrasonic pulsed Doppler technique is the most promising way to achieve this non-invasively. We have investigated mitral regurgitation using a combination of the ultrasonic pulsed Doppler technique and ultrasonic two dimensional echocardiography, and have reported that the overall direction of regurgitant flow from the mitral orifice into the left atrial cavity can be shown non-invasively from the distribution of abnormal Doppler signals in the left atrial cavity.

In the present study, we attempted to detect the site of regurgitant flow in the short-axis section of the mitral orifice as well as the overall direction in the long-axis section and to examine correspondence of the site and overall direction of the mitral regurgitant flow with various types of underlying valve lesions.

(1) OVERALL DIRECTION OF REGURGITANT FLOW INTO LEFT ATRIAL CAVITY

The detection rate of the overall direction of regurgitant flow into the left atrial cavity was low in the preliminary report, ${ }^{6}$ but it reached $70 \%$ in the present work. The main reason for this appeared to be the improvement in detection techniques, including adjustment of equipment and more thorough examination over the left atrial cavity.

The overall direction of regurgitant flow was classified into three directions, that is (a) toward the anterior portion of the left atrial cavity, (b) toward the posterior portion, and (c) toward the central portion or spreading widely over the entire left atrial cavity. In the present study, "regurgitant flow toward the anterior" meant that which was detected not only in the anterior portion of the left atrial cavity, but also along the anterior wall of the left atrium. Regurgitant flow toward the anterior portion of the left atrial cavity was always found in cases of prolapse of ruptured chordae tendineae of the posterior mitral leaflet. The majority of patients with regurgitant flow directed to the posterior portion of the left atrial cavity had prolapse of the anterior mitral leaflet, including a case of rheumatic valvular disease with a dislocation of the anterior leaflet. Most of the patients with regurgitant flow directed toward the central portion of the left atrial cavity had rheumatic valvular disease.

In cases of mitral regurgitation caused by prolapse, the direction of the regurgitant flow into the left atrial cavity differed depending on whether the changes were in the anterior leaflet or the posterior leaflet; thus it is possible that diagnosis of the damaged site of the leaflets can be made from the direction of the regurgitant flow.

In cases of mitral regurgitation caused by rheumatic valvular disease, the regurgitant flow was mainly toward the central portion of the left atrial cavity. The rheumatic changes usually affect both leaflets, so that the regurgitant flow is mainly directed to the central portion of the left atrial cavity. Thus, we conclude that the direction of the regurgitant flow differs depending upon the underlying condition.

The clinical value of the combined use of the ultrasonic Doppler technique and two dimensional echocardiography is considered to be confirmed by the satisfactory agreement between the direction of regurgitant flow determined by the Doppler method and that by left ventriculography. In a case with ruptured chordae tendineae of the anterior mitral valve, however, the direction of regurgitant flow detected by the two methods did not agree. In this case, the ruptured chordae reversed into the central portion of the left atrial cavity in systole. The Doppler signals resulting from the vibration of the ruptured chordae tendineae might be misunderstood as being caused by the regurgitant flow. Care should be taken, in general, with such conditions using the Doppler technique.

\section{(2) SITE OF REGURGITATION IN MITRAL ORIFICE}

The regurgitant flow signals were localised near the anterolateral commissure, in the mid-part, or near the posteromedial commissure of the mitral orifice depending on the patient. In cases of mitral valve prolapse, the site of regurgitant flow detected by the Doppler method agreed well with the site of valve lesions confirmed during operation. Using this technique, it appears possible to be sure of the site of the regurgitation in the mitral orifice non-invasively.

In three cases, the localisation of regurgitation detected by the Doppler method was not the same as the site of the valvular lesion. The reason for this is not known, but possibly the section plane did not precisely involve the short axis, but was oblique to the mitral ring 
so that the entire mitral orifice was not satisfactorily imaged by this section.

It was shown in the present study that the overall direction and site of regurgitation in the mitral valve are closely related to the conditions of the underlying valve lesion. Therefore, the information on the direction and site of mitral regurgitation may be useful in the preoperative estimation of the mitral valve lesion. So far, left ventriculography has offered little information on the site of regurgitation in the valve. The present method, however, can offer direct information on the site of regurgitation in the valve.

Thus, the ultrasonic pulsed Doppler technique combined with two dimensional echocardiography should be useful for the pre- and postoperative evaluation for mitral valve repair and open mitral commissurotomy, as well as in the pathophysiological analysis of mitral regurgitation.

\section{References}

1 Peronneau P, Xhaard M, Nowicki A, Pellet $M$, Delouche $\mathrm{PH}$, Higlais J. Pulsed Doppler ultrasonic flow meter and flow pattern analysis. In: Roberts C, ed. Blood flow measurement. London: Sector Publishing, 1972: 24-8.

2 Lorch GS, Rubenstein SA, Baker DW, Dooley T, Dodge HT. Doppler echocardiography. Use of a graphical display system. Circulation 1977; 56: 576-85.
3 Kalmanson D, Veyrat C, Bouchareine F, Degroote A. Non-invasive recording of mitral valve flow velocity patterns using pulsed Doppler echocardiography. Application to diagnosis and evaluation of mitral valve disease. Br Heart f 1977; 39: 517-28.

4 Johnson SL. Pulsed-Doppler echocardiography. Localization of murmurs and spectrum of clinical applications. In: Mason DT, ed. Advances in heart diseases. Vol 1. New York: Grune \& Stratton, 1977: 389-418.

5 Abbasi AS, Allen MW, DeCristofaro D, Ungar I. Detection and estimation of the degree of mitral regurgitation by range-gated pulsed Doppler echocardiography. Circulation 1980; 61: 143-7.

6 Miyatake K, Kinoshita N, Nagata S, et al. Intracardiac flow pattern in mitral regurgitation studied with combined use of the ultrasonic pulsed Doppler technique and crosssectional echocardiography. Am 7 Cardiol 1980; 45: 15562.

7 Feigenbaum H. Echocardiography. 2nd ed. London: Henry Kimpton, 1976.

8 Wann LS, Feigenbaum H, Weyman AE, Dillon JC. Crosssectional echocardiographic detection of rheumatic mitral regurgitation. Am f Cardiol 1978; 41: 1258-63.

Requests for reprints to Dr Kunio Miyatake, National Cardiovascular Center, 125 Fujishiro-dai 5-chome, Suita, Osaka 565, Japan. 